\title{
Contact Anosov flows on hyperbolic 3-manifolds
}

\author{
PATRICK FOULON \\ BORIS HASSELBLATT
}

Geodesic flows of Riemannian or Finsler manifolds have been the only known contact Anosov flows. We show that even in dimension 3 the world of contact Anosov flows is vastly larger via a surgery construction near an $E$-transverse Legendrian link that encompasses both the Handel-Thurston and Goodman surgeries and that produces flows not topologically orbit equivalent to any algebraic flow. This includes examples on many hyperbolic 3-manifolds, any of which have remarkable dynamical and geometric properties.

To the latter end we include a proof of a folklore theorem from 3-manifold topology: In the unit tangent bundle of a hyperbolic surface, the complement of a knot that projects to a filling geodesic is a hyperbolic 3-manifold.

37D20; 57N10, 57M50

Dedicated to the memory of William P Thurston

\section{Introduction}

\subsection{Summary of the main results}

Theorems 1.6 and 1.9 are our main results, and the essence of their conclusions is summarized as follows:

Theorem 1.1 There are contact Anosov flows that are not topologically orbit equivalent to algebraic flows; this includes examples on numerous hyperbolic 3-manifolds.

Here we use the following definition.

Definition 1.2 (Katok and Hasselblatt [38]) Let $M$ be a manifold and $\varphi: \mathbb{R} \times M \rightarrow$ $M$ be a smooth flow with generating vector field $X$. Then $\varphi$ is said to be an Anosov flow if the tangent bundle $T M$ splits as $T M=E^{\varphi} \oplus E^{+} \oplus E^{-}$(the flow, strongunstable and strong-stable directions, respectively), where $E^{\varphi}=\mathbb{R} X \neq\{0\}$, in such a way that there are constants $C>0$ and $\eta>1>\lambda>0$ such that

$$
\left\|D \varphi^{-t} \uparrow E^{+}\right\| \leq C \eta^{-t} \quad \text { and } \quad\left\|D \varphi^{t} \uparrow E^{-}\right\| \leq C \lambda^{t}
$$


for $t>0$. The weak-unstable and weak-stable bundles are $E^{\varphi} \oplus E^{+}$and $E^{\varphi} \oplus E^{-}$, respectively. $\varphi$ is said to be a contact Anosov flow if there is a smooth invariant 1-form $A$ such that $A \wedge d A \wedge \cdots \wedge d A$ defines a (clearly smooth invariant) volume.

A well-known consequence of this definition is that $E^{ \pm}$are tangent to foliations $W^{ \pm}$ with smooth leaves.

Remark 1.3 If $\omega$ is a form invariant under an Anosov flow, then so is $i_{X} \omega$, where $X$ is the generating vector field. Volume-preserving Anosov flows are ergodic, i.e., bounded invariant functions are constant. This implies that $A(X)=$ const so, up to constant rescaling of $X$, a contact Anosov flow is the Reeb flow of the contact form.

A bounded invariant form restricts to 0 on the stable bundle (see Feres and Katok [19, Lemma 1], and Kanai [36, page 221f]) and the unstable bundle. Thus, a bounded invariant 1-form for an Anosov flow is constant on $X$ and vanishes on $E^{+} \oplus E^{-}$, so a contact Anosov flow on a 3-manifold can be described as the Reeb flow of a contact form whose contact structure is of the form $E^{+} \oplus E^{-}$for line bundles satisfying (1). ${ }^{1}$

Following Handel and Thurston [28] and Tomter [51] we use the following definition.

Definition 1.4 An Anosov flow on a 3-manifold is said to be of algebraic type if it is finitely covered by the geodesic flow of a surface or the suspension of a diffeomorphism of the 2-torus.

Theorems 1.6 and 1.9 also provide remarkable properties of the resulting flows such as the existence of distinct isotopic closed orbits.

We include a proof of Theorem 1.12, a needed folklore theorem from 3-manifold topology: In the unit tangent bundle of a hyperbolic surface, the complement of a knot that projects to a filling geodesic is a hyperbolic manifold.

\subsection{Algebraic and anomalous Anosov flows}

As mechanical systems amenable to statistical analysis (see Artin [1] and Hopf [34]), geodesic flows of manifolds of negative curvature have been a classical and central proving ground in hyperbolic dynamics. That they preserve a natural smooth measure is one of the consequences of their Hamiltonian nature, but it can also be seen as a consequence of the fact that they preserve a natural contact form $p d q$.

\footnotetext{
${ }^{1}$ That contact structure is tight: the Reeb flow of an overtwisted contact structure has a contractible orbit (see Hofer [33, Theorem 1]) while an Anosov flow of a 3-manifold does not (see Plante and Thurston [46] and Barbot [5, page 18]).
} 
Remark 1.5 While every Anosov flow admits a canonical invariant 1-form (defined by being 1 on the generating vector field and having its kernel spanned by the strong stable and unstable subspaces), this is only rarely smooth (see Plante [45, Theorem 4.7] and Foulon and Hasselblatt [21]), and for suspensions, this canonical 1-form is smooth but closed, so $A \wedge d A \wedge \cdots \wedge d A=0$; the extreme opposite of the contact case. Thus, the contact condition can be viewed as a constraint on the canonical 1-form.

For the discrete-time counterpart, Anosov diffeomorphisms, all known examples are topologically equivalent to a linear representative, and thereby the known situations are classified by a list of algebraic objects. Anosov flows are not this rigid (see Franks and Williams [22], Christy [13], Handel and Thurston [28] and Goodman [26]). However, some experts thought that surgeries could not produce contact flows, and the published record (see, for example, Hamenstädt [27], Foulon [20], Liverani [39] and Fang [14]) reflects a paucity of examples. We remark that Anosov flows on the unit tangent bundle of a surface are always topologically orbit equivalent to an algebraic flow (see Ghys [24]). The present work (announced in Foulon [20]) presents a wealth of genuinely new contact Anosov flows, showing that the universe of contact flows is vastly greater than that of geodesic flows of Riemannian or Finsler manifolds. A separate aspect of interest is that our surgery is carried out along a Legendrian knot, not necessarily near a periodic orbit (see also Bonatti and Langevin [10]). Our context is one that makes many such knots available, and, on the other hand, for any one such knot we describe entire families of surgeries.

That contact Anosov flows need not be topologically orbit equivalent to an algebraic flow means there are no "shortcuts" in the study of their structure. For instance, the proof that smoothness of the invariant foliations implies smooth, hence in particular topological, conjugacy to a standard algebraic system, up to a canonical time-change (see Benoist, Foulon and Labourie [8; 9]) had to laboriously exclude the Lie-algebraic structures that do not fit into that collection of classical examples. It would have been greatly simplified by knowing a priori that contact flows are topologically orbit equivalent to an algebraic flow; we show that there is no such shortcut.

By structural stability, connected components of the collection of contact flows (in the $C^{1}$-topology) lie in orbit equivalence classes. All previously known orbit equivalence classes contain an algebraic representative, so Theorem 1.1 (and Theorems 1.6 and 1.9 below) can be viewed as describing connected components of altogether new types.

\subsection{The main result}

While Theorem 1.1 is our essential conclusion, there is additional value both in the remarkable properties of the examples obtained when starting with a Legendrian knot 
having hyperbolic complement and in the method: a surgery that produces many new examples of volume-preserving Anosov flows on 3-manifolds and, upon a time-change, new examples of contact Anosov flows, and which subsumes the Handel-Thurston surgery [28] and the Goodman surgery [26] (for contact flows; Goodman surgery was invented to deal with suspensions of toral automorphisms).

Theorem 1.6 For a contact Anosov flow on a 3-manifold $M$ and an $E$-transverse Legendrian knot (Definition 2.1) in $M$, there is a family of smooth Dehn surgeries that produce new contact Anosov flows.

If the original contact flow is the geodesic flow on the unit tangent bundle of a negatively curved surface, then these surgeries include the Handel-Thurston surgery, in which case the resulting flow has the following properties:

(1) It acts on a manifold that is not a unit tangent bundle.

(2) It is not topologically orbit equivalent to an algebraic flow.

(3) Its weak stable foliation is not transversely projective (Barbot [2, Théorème A]).

(4) Its Anosov splitting $T M=E^{\varphi} \oplus E^{+} \oplus E^{-}$is not $C^{1+\text { zygmund }}$, that is, does not have "little Zygmund" (hence not Lipschitz-continuous) derivative (Theorem 1.7).

(5) Its topological and volume entropies differ, or, equivalently, the measure of maximal entropy is always singular (Theorem 1.8).

Theorem 1.7 (Hurder and Katok [35], Ghys [25]) If a volume-preserving Anosov flow on a 3-manifold has $C^{1+\text { zygmund }}$ Anosov splitting, then it is smoothly conjugate to a geodesic flow.

Theorem 1.8 (Foulon [20]) Contact Anosov flows on 3-manifolds for which the topological and Liouville entropies coincide are up to finite covers smoothly conjugate to a geodesic flow of constant curvature.

We now obtain contact Anosov flows on hyperbolic manifolds.

Theorem 1.9 There are contact Anosov flows on hyperbolic manifolds: If the complement in $M$ of the $E$-transverse Legendrian knot in Theorem 1.6 is a hyperbolic manifold, then all but finitely many of our Dehn surgeries produce a hyperbolic manifold (and hence neither the unit tangent bundle of a surface nor a torus bundle over a circle). The resulting contact Anosov flow (and any contact Anosov flow topologically orbit equivalent to it) has the following properties.

(1) (1)-(5) of Theorem 1.6. 
(2) It is not quasigeodesic.

(3) Its orbits are geodesics for suitable Riemannian metrics on $M$.

(4) Each closed orbit is freely homotopic to infinitely many others ${ }^{2}$, and each free homotopy class is an isotopy class (see Fenley [15, Theorem A], Barthelmé [6, Remark 5.1.16, Theorem 5.3.3], and Barthelmé and Fenley [7]).

(5) Each closed orbit is related to at most finitely many others by the pair being the boundary of an embedded cylinder ${ }^{3}$ [7].

(6) It is associated with a new example of a quasigeodesic pseudo-Anosov flow (see Definition 1.10, Fenley [17] and Thurston [48, Section 5]).

Definition 1.10 A quasigeodesic curve is one that is efficient, up to a bounded multiplicative distortion, in measuring distances in relative homotopy classes, and a flow is said to be quasigeodesic if all flow lines are quasigeodesics (see Fenley [16]).

Remark 1.11 Theorem 1.9 (4) says that there is no knot theory of closed orbits within a free homotopy class ${ }^{4}$. One might ask about the growth rate (as a function of period) of the number of closed orbits in a given isotopy class.

The folklore theorem that gives substance to Theorem 1.9 is the following:

Theorem 1.12 Suppose $\Sigma$ is a hyperbolic surface, $\pi: M:=S \Sigma \rightarrow \Sigma$ its unit tangent bundle, $\gamma: S^{1} \rightarrow M$ continuous such that $\mathfrak{c}:=\pi \circ \gamma$ is an indivisible filling closed geodesic in $\Sigma$.Then $S \Sigma \backslash\left(\gamma\left(S^{1}\right)\right)$ is a hyperbolic manifold.

We apply this to the curve of vectors orthogonal to a filling geodesic in $\Sigma$.

Definition 1.13 A closed curve $c$ in a surface fills the surface if $\alpha \cap c \neq \varnothing$ whenever $\alpha$ is a closed curve that is not null-homotopic. It is said to be indivisible if it is not the same geodesic traversed more than once.

Remark 1.14 This paper originally contained Theorem 1.12 with a flawed proof we were unable to fix, so the submitted version omitted it. We are grateful to Danny Calegari for suggesting the proof on his blog [12]. Theorem 1.12 appears to be a folklore result in 3-manifold theory, and the experts we consulted found it easier to prove this result than to locate a proof in the literature. Appendix B presents a proof that reflects suggestions by Calegari, Fenley, Otal and Walsh.

\footnotetext{
${ }^{2}$ For algebraic flows, free homotopy classes of closed orbits have cardinality at most 2.

${ }^{3}$ This relation is neither transitive nor reflexive. For comparison, isotopy is the equivalence relation of being the boundary components of an immersed cylinder.

4 ... which is the proper setting — analogously to the classical setting for knot theory, $S^{3}$, where knots are null-homotopic
} 
Remark 1.15 The Handel-Thurston examples are not topologically orbit equivalent to a standard model. As the introduction to Handel and Thurston [28] explains:

The standard examples of Anosov flows on three-dimensional manifolds are the geodesic flows on surfaces of negative curvature, and the suspensions of Anosov diffeomorphisms of $T^{2}$.

Both of these families are algebraic; i.e., they fit into the broader category of one-parameter subgroups acting on homogeneous spaces. More precisely, a flow $\phi_{t}$ on a compact connected manifold $M$ is algebraic if $M=\Gamma \backslash G / K$, where $G$ is a Lie group, $K$ is a compact subgroup, and $\Gamma$ is a discrete cocompact subgroup of $G$ acting by left multiplication; the flow is given by $\phi_{t}: \Gamma g K \mapsto \Gamma g(\exp t \alpha) K$ where $\alpha$ is an element of the Lie algebra of $G$.

Tomter showed that if $M$ is three dimensional and if $\phi_{t}$ is an algebraic Anosov flow, then some finite cover of $M$ is either a geodesic flow or the suspension of a toral diffeomorphism [T] (Tomter called these flows $(G, \Gamma)$-induced). By examining the fundamental groups of the manifolds we construct, we show that our examples are not algebraic. These are the first such examples.

Since Handel and Thurston obtain their conclusion by examining the fundamental group, their examples are not topologically orbit equivalent to any algebraic flow. Likewise, those of our examples whose phase space is a hyperbolic manifold are not algebraic in this sense. Indeed, Handel and Thurston remark that "it would be very interesting to find examples of Anosov flows... on an atoroidal $M$ ". (Note that "hyperbolic" implies "atoroidal".) This was accomplished by Goodman [26], and we show how to do so in the contact category.

The first author has previously presented this construction in lectures, and this is reflected in the literature (for example, Barbot [5, pages 53 and 75; 3, page 754], Fang [14, page 1191], Hasselblatt [29, page 254], Mitsumatsu [42, page 1417]).

\subsection{Methods}

Both Handel-Thurston and Goodman were inspired by the idea of Dehn surgery. It is of particular interest to point to Goodman [26, page 307], which describes something along rather similar lines to what we do here, but starting from a suspension (of $\left(\begin{array}{ll}2 & 1 \\ 1 & 1\end{array}\right): \mathbb{T}^{2} \rightarrow \mathbb{T}^{2}$ ), a situation complementary to contact flows. In that context the orbit of the origin is a knot with hyperbolic complement, which provides the base for obtaining Anosov flows on hyperbolic manifolds. In our context, there is an orbit that can play an analogous role, once we pass to an isotopic Legendrian perturbation.

The Handel-Thurston examples are graph manifolds, that is, they are obtained by glueing Seifert fibered spaces together along their $\mathbb{T}^{2}$ boundary components. 
The method for showing that this surgery yields contact flows is new: It involves a suitable deformation of the contact form and a time-change adapted to this deformation. Showing that the surgered and time-changed flows are hyperbolic was essentially done in Handel and Thurston [28] and Goodman [26], but we outline a proof by Thierry Barbot that is remarkably clean, direct and transparent [5, page 54].

The construction of contact structures is not our claim to novelty; there are contact structures on any 3-manifold (see Martinet [40]). We give new manifolds with a contact form whose Reeb flow is a nonstandard Anosov flow.

\subsection{Structure of this paper}

Below we prove Theorem 1.9. Section 2 describes the local structure near the knot used to define the surgery, which is described in Section 3. Section 4 shows that this yields contact Anosov flows. Section 5 explains how our surgery subsumes and extends the surgeries of Handel-Thurston and Goodman; it serves to convey that this surgery provides an abundance of examples. Section 6 recalls that in the Handel-Thurston situation we get nonalgebraic flows. Appendix A translates the Alexeev cone criterion for hyperbolicity into the language of Lyapunov-Lorentz metrics, and Appendix B establishes the folklore Theorem 1.12 about hyperbolic knot complements..

\subsection{Contact Anosov flows on hyperbolic manifolds: proof of Theorem 1.9}

Although we describe it in terms of an annulus, our surgery can also be viewed as the deletion of a knot (or tubular neighborhood thereof) followed by what is called a Dehn filling, that is, the glueing-in of a solid torus subject to a prescribed identification of a meridian on the boundary of the knot complement with a curve on the boundary of the inserted torus. The effect of this surgery is described uniquely by the slope of the boundary curve. Therefore, these surgeries are parameterized by $\mathbb{Q} \cup\left\{\frac{1}{0}\right\}$. If the initial knot can be chosen so as to have a hyperbolic complement, then the abundance of hyperbolic manifolds arising from our construction is due to the following result of Thurston's.

Theorem 1.16 (Thurston [49, Theorem 5.8.2; 50], Petronio and Porti [44]) For all but finitely many slopes, Dehn filling a hyperbolic 3-manifold gives rise to a hyperbolic manifold. ${ }^{5}$

Some orbits of contact Anosov flows on hyperbolic manifolds are quite far from being globally minimizing (Theorem $1.6(2)$ ). This follows from

\footnotetext{
5 ... which is Haken for at most finitely many slopes.
} 
Theorem 1.17 (Fenley [15, Theorem B; 16, Theorem A]) Contact Anosov flows on hyperbolic manifolds are not quasigeodesic.

This contrasts with item (3) in Theorem 1.9: contact flows are "geodesible."

Proposition 1.18 If $X$ generates a flow that preserves a contact form $A$, and $g$ is a Riemannian metric with $X \perp \operatorname{ker} A$ and $g(X, X) \equiv 1$, then $X$-orbits are geodesics for $g$.

Remark 1.19 This holds in the hyperbolic case, which is not quasigeodesic. Thus, the metrics in Proposition 1.18 are not negatively curved in this case.

Proof To verify the geodesic equation $\nabla_{X} X=0$, where $\nabla$ is the Levi-Cività derivative, we check $g\left(\nabla_{X} X, \xi\right)=0$ for any vector field $\xi$. Compute the Lie derivative

$$
\mathcal{L}_{X} g(X, \xi)=\nabla_{X} g(X, \xi)=g\left(\nabla_{X} X, \xi\right)+g\left(X, \nabla_{X} \xi\right) .
$$

Since $g(X, \xi)=A(\xi)$, this agrees with

$$
\mathcal{L}_{X} A(\xi)=A([X, \xi])=A\left(\nabla_{X} \xi-\nabla_{\xi} X\right)=g\left(X, \nabla_{X} \xi\right)-g\left(X, \nabla_{\xi} X\right) .
$$

Therefore,

$$
g\left(\nabla_{X} X, \xi\right)=-g\left(X, \nabla_{\xi} X\right)=-\frac{1}{2} \nabla_{\xi} g(X, X)=-\frac{1}{2} \nabla_{\xi} 1=0 .
$$

Finally, a contact flow is $\mathbb{R}$-covered (see Barbot [4, Theorem A]) and on an atoroidal manifold has a transverse regulating pseudo-Anosov flow (see Calegari [11, Corollary 5.3.16]), which is quasigeodesic (see Fenley [18, Theorem 6.1, Section 7]). This proves item (6) of Theorem 1.9.

\section{Local structure near Legendrian knots}

Definition 2.1 A Legendrian curve in a contact manifold is a curve tangent to the contact structure at every point. In the presence of a contact Anosov flow, a Legendrian curve (which is by construction transverse to the flow) is said to be $E$-transverse if it is also transverse to both the strong stable and strong unstable subbundles $E^{-}$and $E^{+}$of the flow.

Proposition 2.2 Suppose $\varphi$ is a contact Anosov flow with orientable strong-stable subbundle $E^{-}$and $\gamma$ is an $E$-transverse Legendrian knot. 
Then there is a smooth annular transversal $\Sigma$ through $\gamma$ that is transverse to $E^{-}$and $E^{+}$away from $\gamma$, and there are coordinates $(s, w)$ on $\Sigma$ such that $s \in S^{1}$ is the parameter for $\gamma$, and on $\Lambda:=\bigcup_{t \in(-\eta, \eta)} \varphi^{t}(\Sigma)$ the contact form $A$ satisfies

$$
A=d t+w d s, \quad d A=d w \wedge d s \quad \text { and } \quad A \wedge d A=d t \wedge d w \wedge d s .
$$

Here, $t$ denotes the transverse parameter given by the flow.

Remark 2.3 This in particular implies that $\Sigma$ is transverse to the flow, which means that our surgery does indeed reconnect orbits-without transversality it would not even be clear whether orbits become smooth curves after surgery.

Proof For a Legendrian knot $\gamma: S^{1} \rightarrow M, s \mapsto \gamma(s)$ consider transversals

$$
T^{-}:=\bigcup_{s \in S^{1}} W_{\epsilon}^{-}(\gamma(s)) \quad \text { and } \quad T^{+}:=\bigcup_{s \in S^{1}} W_{\epsilon}^{+}(\gamma(s))
$$

swept out by local stable and unstable manifolds of size $\epsilon>0$. ker $A$ is the common tangent plane of $T^{+}$and $T^{-}$at points of $\gamma$, and $T^{+}$and $T^{-}$are fibered by smooth Legendrian curves (stable and unstable leaves) transverse to $\gamma$.

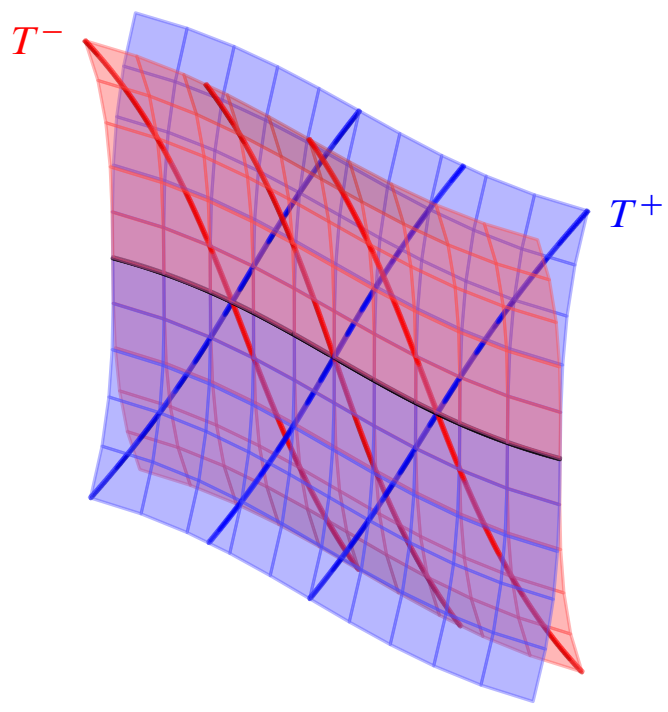

Figure 1: Transversals

Using, for instance, local exponential maps centered at $\gamma(s)$ and a partition of unity we obtain a smooth convex combination $\Sigma$ of these transversals that contains $\gamma$ such that:

- $T_{\gamma(s)} \Sigma=\operatorname{ker} A$ for all $s \in S^{1}$, 
- $\quad \Sigma$ is transverse to both $E^{-}$and $E^{+}$off $\gamma$,

- $\Sigma$ is smoothly fibered by Legendrian curves transverse to $\gamma$.

Next, we define convenient coordinates on $\Sigma$. One coordinate on $\Sigma$ is the parameter $s \in S^{1}$ of the Legendrian knot $\gamma$, another is a smoothly chosen parameter $v$ along the Legendrian curves transverse to $\gamma$. In these coordinates

$$
A=d t+f(s, v) d s .
$$

Since $\gamma$ is Legendrian, $A_{(0, s, 0)}(\partial / \partial s)=0$, so $f(s, 0)=0$, while,

$$
0 \neq d A=(\partial / \partial v) f(s, v) d v \wedge d s,
$$

so $(\partial / \partial v) f(s, v) \neq 0$. Thus, the transformation

$$
(s, v) \mapsto(s, f(s, v))=:(s, w)
$$

is nonsingular and gives coordinates on $\Sigma$ that are as desired.

\section{Contact surgery}

The surgeries we describe in this work are topologically of the Dehn filling type: One can view them as removing a tubular neighborhood of a knot and glueing that solid torus back in such a way as to match a prescribed closed curve on the boundary with a meridian. The surgery is then described by the slope of the prescribed curve, a pair of coprime integers. For a pair $(1, n)$ this can equivalently be described as splitting apart an annulus in the manifold and glueing both copies of this annulus back together with a shear, that is, by again identifying the 2 annuli via the shear map. We remark that although this shear map is a homeomorphism of the annulus, this defines a discontinuous operation since the resulting space is no longer homeomorphic to the original one. We perform surgery of the latter type, but have occasion to keep in mind the knot near which the surgery takes place.

Moreover, it is important for us that the surgery yield a smooth manifold, not just a topological one, and moreover, that the vector field that generates the flow gives rise to a well-defined vector field on the surgered manifold.

Proposition 2.2 gives an annulus that contains the Legendrian knot $\gamma$ and is uniformly transverse to the flow. (By moving $\gamma$ along the flow one obtains a homotopic knot $\gamma^{\prime}$ with a tubular neighborhood whose boundary contains this annulus; this tubularknot-neighborhood point of view will prevail in Section 6, where we study topological properties.) We split the flow-box chart from Proposition 2.2 into 2 one-sided flow-box 
neighborhoods of the surgery annulus, and while the initial transition map between these on $\{0\} \times S^{1} \times(-\epsilon,+\epsilon)$ is the identity, the surgered manifold is defined by imposing the desired shear as the transition map on this annulus. The use of flow-box charts ensures that the original vector field defining the contact Anosov flow defines a smooth vector field on the surgered manifold, that is, that the orbits are reglued to smooth curves.

The transition map pulls meridians around the equator $q \in \mathbb{N}$ times before exiting (see Figure 2).

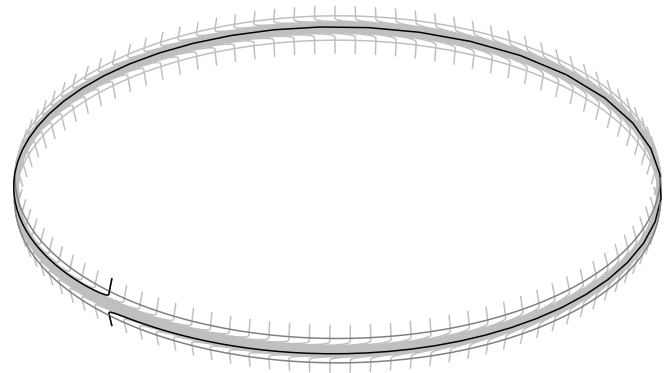

Figure 2: Surgery in the chart (here, $q=1$ )

Definition 3.1 The contact $(1, q)$-Dehn surgery for $q \in \mathbb{N}$ is defined by imposing on the aforementioned chart overlap the transition map

$$
F: S^{1} \times(-\epsilon, \epsilon) \rightarrow S^{1} \times(-\epsilon, \epsilon), \quad(s, w) \mapsto(s+f(w), w) .
$$

Here (see Figure 3),

$$
f:[-\epsilon, \epsilon] \rightarrow S^{1}, \quad w \mapsto \exp (-i q g(w / \epsilon)),
$$

where $g: \mathbb{R} \rightarrow[0,2 \pi]$ is a monotone smooth function with $0 \leq g^{\prime} \leq 4$ even such that $g((-\infty,-1])=\{0\}$ and $g([1, \infty))=\{2 \pi\}$ (see Figure 3$)$.
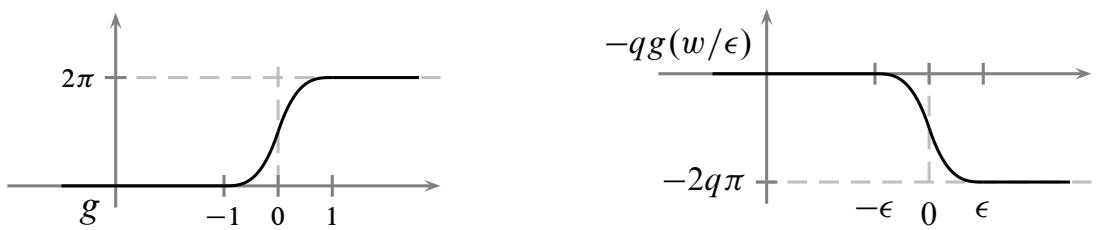

Figure 3: The function $g$

Because of our use of flow-box charts it is apparent that the vector field generating the original contact Anosov flow defines a smooth vector field $X$ on the surgered manifold 
$M$. The original contact form induces a contact form on the surgered manifold only if the coordinate representation $A=d t+w d s$ is preserved by the transition map $F$ between the two overlapping charts that define the surgery. Unfortunately, this is not so:

Lemma 3.2 $F_{*} A=A+w f^{\prime}(w) d w$ and $F_{*} d A=d A$.

Proof Since $d(s+f(w))=d s+f^{\prime}(w) d w$ we have

$$
\begin{gathered}
F_{*} A=d t+w d(s+f(w))=A+w f^{\prime}(w) d w \\
F_{*} d A=d w \wedge d(s+f(w))=d w \wedge d s=d A .
\end{gathered}
$$

This completes the proof.

This implies that $d A$ induces a well-defined (albeit not necessarily exact) 2-form on the surgered manifold. Moreover, since $F_{*} d t=d t$, we see that $A \wedge d A=d t \wedge d A$ is well-defined on the surgered manifold. Indeed:

Corollary 3.3 The new flow preserves the Liouville volume defined by $A \wedge d A$.

We remark that Handel and Thurston also obtained volume-preserving flows.

\section{Contact Anosov property}

We produce a contact form on the surgered manifold by giving representations $A_{-}$ and $A_{+}$for it on the 2 one-sided flow-box charts (whose overlap defines the surgery annulus) that on one hand agree with $A=d t+w d s$ near the chart boundary and on the other hand are such that $F_{*} A_{-}=A_{+}$. This follows from the existence of a function $h$ such that $F_{*}(A-d h)=A+d h$ : deforming $A$ to $A-d h$ on one side of the surgery and to $A+d h$ on the other gives a contact form that glues together nicely, and $d A$ is unchanged. To define $h$ recall from Lemma 3.2 that $F_{*} A=A+w f^{\prime}(w) d w$ and "split the difference": To get $d h=\frac{1}{2} w f^{\prime}(w) d w$ at points where $F \neq$ Id set

$$
h(t, w):=\frac{1}{2} \lambda(t) \int_{-\epsilon}^{w} x f^{\prime}(x) d x
$$

on $(-\eta, \eta) \times(-\epsilon, \epsilon)$ and $h=0$ outside. Here, $\lambda: \mathbb{R} \rightarrow[0,1]$ is a smooth bump function

- supported in $(-\eta, \eta)$,

- with $\lambda^{-1}(\{1\})$ an interval containing a neighborhood of 0 ,

- monotone on the two intervals where it takes intermediate values, 


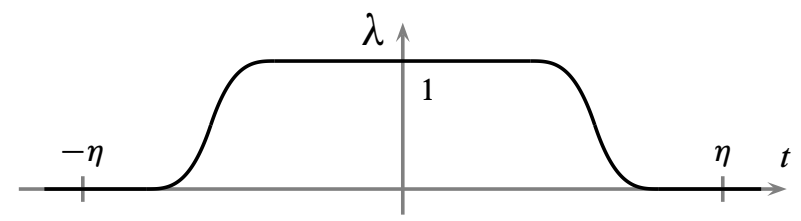

Figure 4: The function $\lambda$

- with $\left|\lambda^{\prime}\right| \leq \pi / \eta$.

Equation (3) defines a smooth function on $M$ since $h=0$ for $t$ near $\pm \eta$ by choice of $\lambda$.

Lemma 4.1 If $0<\epsilon<\frac{\eta}{2 \pi q}$, then $|d h(X)|<1$.

Proof Clear where $d h=0$. Elsewhere use $\left|f^{\prime}(w)\right|=q\left|\frac{d}{d w} g\left(\frac{w}{\epsilon}\right)\right|=\frac{q}{\epsilon}\left|g^{\prime}\left(\frac{w}{\epsilon}\right)\right|$, $\left|\lambda^{\prime}\right| \leq \pi / \eta, 0 \leq g^{\prime} \leq 4$ to get $\left|\frac{\partial h}{\partial t}\right|=\left|\frac{1}{2} \lambda^{\prime}(t) \int_{0}^{w} x f^{\prime}(x) d x\right| \leq\left|\frac{q \pi}{2 \eta}\right| \int_{0}^{\epsilon} x \frac{\left|g^{\prime}\right|}{\epsilon} d x \leq\left|\frac{q \pi}{2 \eta} \epsilon \frac{4}{\epsilon} \int_{0}^{\epsilon} d x\right|=\frac{2 q \pi}{\eta} \epsilon<1$.

This completes the proof.

Theorem 4.2 The Dehn surgery of Definition 3.1 near a Legendrian knot and a choice of bump function as in (3) yield a contact flow $\varphi_{h}^{t}$ defined by a vector field $X_{h}:=\frac{X}{1 \pm d h(X)}$, where the contact form is $A_{h}:=A \pm d h$. Here, for both $X_{h}$ and $A_{h}$, we take "+" on one chart and "-" on the other.

Proof $h$ is independent of $s$, so $F_{*} h=h$, hence $F_{*} d h=d h$. That $A_{h}$ is smooth is clear away from the surgery. At the surgery it follows since Lemma 3.2 yields

$$
F_{*}(A-d h)=F_{*} A-F_{*} d h=(A+2 d h)-F_{*} d h=A+d h .
$$

Next, $A_{h}$ is clearly a contact form where $d h=0$. Elsewhere,

$$
\begin{aligned}
A_{h} \wedge d A_{h}=(A \pm d h) \wedge d(A \pm d h) & =(A \pm d h) \wedge d A \\
& =\left(A \pm\left(\frac{\partial h}{\partial t} d t+\frac{\partial h}{\partial w} d w\right)\right) \wedge d A=\left(1 \pm \frac{\partial h}{\partial t}\right) d t \wedge d A
\end{aligned}
$$

by Lemma 5.1 , and $1 \pm \frac{\partial h}{\partial t} \neq 0$ by Lemma 4.1 .

$X_{h}$ is a smooth vector field since by Lemma 4.1 the denominator is nonzero, and " \pm " matches smoothly on the surgery annulus, near which $d h(X)=\partial h / \partial t=0$. 
Finally, the flow $\varphi_{h}^{t}$ generated by $X_{h}$ preserves $A_{h}$ since

$$
A_{h}\left(X_{h}\right)=A_{h}\left(\frac{X}{1 \pm d h(X)}\right)=\frac{(A \pm d h)(X)}{1 \pm d h(X)}=\frac{1 \pm d h(X)}{1 \pm d h(X)}=1
$$

implies $\mathcal{L}_{X_{h}} A_{h}=0$.

Theorem 4.3 The Dehn surgery of Definition 3.1 near a Legendrian knot with the choices of Theorem 4.2 yields a contact Anosov flow $\varphi_{h}^{t}: M \rightarrow M$.

Remark 4.4 This is effectively proved in Goodman [26] and Handel and Thurston [28]. With kind permission from Thierry Barbot we outline here his elegant and hitherto unpublished version [5, page 54] of the argument.

Proof (Barbot [5]) We use the formulation of hyperbolicity in terms of suitable Lyapunov-Lorentz metrics as described in Proposition A.1; this is a reformulation of the usual cone criterion for hyperbolicity.

By Proposition A.1 there is a pair of Lyapunov-Lorentz metrics for $\varphi^{t}$. We deform these to work as needed for $\varphi_{h}^{t}$. First, we arrange (using a partition of unity, say) for the Lyapunov-Lorentz metrics for $\varphi^{t}$ to have the form

$$
Q^{ \pm}= \pm d w d s-c d t^{2}
$$

in $\Lambda$, where $c$ is chosen sufficiently small to ensure that the positive $Q^{ \pm}$-cone contains $E^{ \pm}$. Choose $\beta: \mathbb{R} \rightarrow \mathbb{R}^{+}$smooth with $\beta((-\infty, 0])=\{1\}, \beta([\eta, \infty))=0$ and $\beta^{\prime}<0$ on $(-\eta, \eta)$ to obtain:

Claim 4.5 Taking $Q_{0}^{ \pm}$and $Q_{1}^{ \pm}$to be the old Lyapunov-Lorentz metrics outside $\Lambda$ and

$$
Q_{i}^{ \pm}:= \pm\left(d w d s+i \beta(t) f^{\prime}(w) d w^{2}\right)-c d t^{2}
$$

inside defines Lyapunov-Lorentz metrics for $\varphi_{h}^{t}$. Here, $i=0$ on one side of the surgery and $i=1$ on the other.

Proof of Claim 4.5 Our choice of $f$ and $\beta$ ensures that these are smooth metrics.

These choices fit together, that is, $F$ sends the choice on one side to that on the other, because for $t=0$ :

$$
\begin{aligned}
F_{*} Q_{0}^{ \pm}=F_{*}\left( \pm d w d s-c d t^{2}\right) & = \pm d w d(s+f(w))-c d t^{2} \\
& = \pm\left(d w d s+f^{\prime}(w) d w d w\right)-c d t^{2} \\
& = \pm\left(d w d s+\beta(0) f^{\prime}(w) d w^{2}\right)-c d t^{2}=Q_{1}^{ \pm} .
\end{aligned}
$$

This completes the proof of Claim 4.5. 
Of the required properties in Proposition A.1, (2) and (3) are clear. We wish to check that properties (1) and (4) in Proposition A.1 are inherited from the same properties for $\varphi^{t}$. Outside of $\Lambda$ this is given since $\varphi=\varphi_{h}$.

On $\Lambda$ we have been using a flow-box chart for $\varphi_{t}$, so the flow is represented by a shift in time. This makes it a $Q_{0}^{ \pm}$-isometry. We need to see how the surgery and the choice of $\beta$ affect invariance, that is, Proposition A.1 (4). To that end it is helpful to restrict attention to the trace of these cones in the $s w$-plane. Here, the $Q_{0}^{ \pm}$-cones show as quadrants since

$$
0=Q_{0}^{ \pm}\left(a \frac{\partial}{\partial s}+b \frac{\partial}{\partial w}\right)=a b
$$

implies $a=0$ or $b=0$. On the immediate other side of the surgery $(t=0)$ they are given by

$$
0=Q_{1}^{ \pm}\left(a \frac{\partial}{\partial s}+b \frac{\partial}{\partial w}\right)=a b+f^{\prime}(w) b^{2}=\left(a+f^{\prime}(w)\right) b,
$$

which implies $a=-f^{\prime}(w) b$ or $b=0$; since $f^{\prime} \leq 0$, this describes a subcone of the first and third quadrant that shares the horizontal axis. Since $\Lambda$ is a flow-box, $\varphi_{h}$ leaves these cones exactly invariant, which means that strict monotonicity of $\beta$ produces a strictly invariant cone field that connects smoothly at $t=\eta$. This gives Proposition A.1 (4).

To obtain Proposition A.1 (1) note that, $\Lambda$ being a flow-box chart,

$$
\pm Q_{1}^{ \pm}\left(D \varphi_{h}^{t}\left(a \frac{\partial}{\partial s}+b \frac{\partial}{\partial w}\right)\right)= \pm Q_{1}^{ \pm}\left(a \frac{\partial}{\partial s}+b \frac{\partial}{\partial w}\right)=a b+\beta(t) f^{\prime}(w) b^{2}
$$

is increasing in $t$. Combined with the exponential growth outside $\Lambda$, this yields Proposition A.1 (1).

\section{Constructing examples}

In this section we describe how our construction subsumes those of Handel and Thurston [28] and Goodman [26] and goes beyond these by considering different initial geodesics as starting points or applying surgeries simultaneously or repeatedly.

\subsection{Handel-Thurston surgery and beyond}

To reproduce the setting used by Handel and Thurston, consider a negatively curved oriented surface and select a closed geodesic $\mathfrak{c}: S^{1} \rightarrow \Sigma, s \mapsto \mathfrak{c}(s)$. If this geodesic is simple and separating, we denote the unit tangent bundles of the two components of the surface by $M_{1}$ and $M_{2}$.

We deform the geodesic flow $g^{t}$ on the unit tangent bundle $S \Sigma$ of the surface to a flow on a new 3-manifold. The common boundary of $M_{1}$ and $M_{2}$ is a torus $S^{1} \times S^{1}$ 


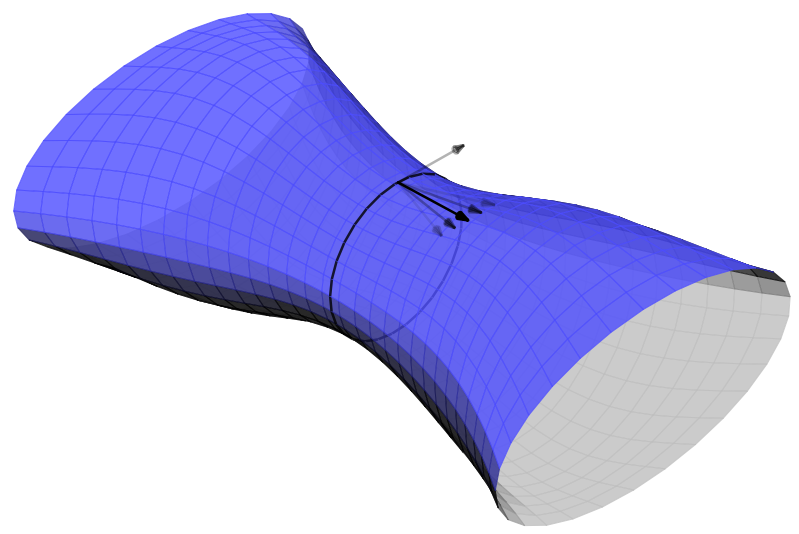

Figure 5: A simple closed geodesic and normal vectors

parametrized by the parameter $s$ of the geodesic $\mathfrak{c}$ and the angle $\theta$ with the tangent vector of the geodesic. The Legendrian knot is the unit vector field perpendicular to $\mathfrak{c}$ given by $\theta=-\pi / 2$.

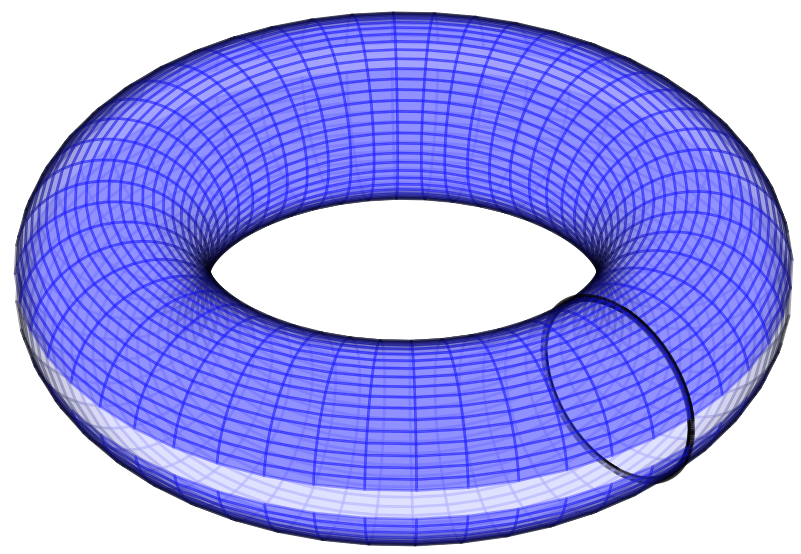

Figure 6: The annulus $S^{1} \times\left(-\frac{\pi}{2}-\epsilon,-\frac{\pi}{2}+\epsilon\right) \subset S^{1} \times S^{1}$ before surgery

Although we localize the surgery in an annulus around $\theta=-\pi / 2$ inside the torus, topologically this is clearly the same as in [28].

To parametrize a neighborhood $\Lambda$ of the perpendicular unit vector field $\gamma$ of $\mathfrak{c}$, linearize the angle $\theta$ with the tangent vector field to $\mathfrak{c}$ by taking $w:=\frac{\ell}{2 \pi} \cos \theta$ for $\theta$ near $-\pi / 2$, where $\ell$ is the length of $\mathfrak{c}$. This gives parameters

$$
(t, s, w) \in \Omega:=(-\eta, \eta) \times S^{1} \times(-\epsilon,+\epsilon),
$$




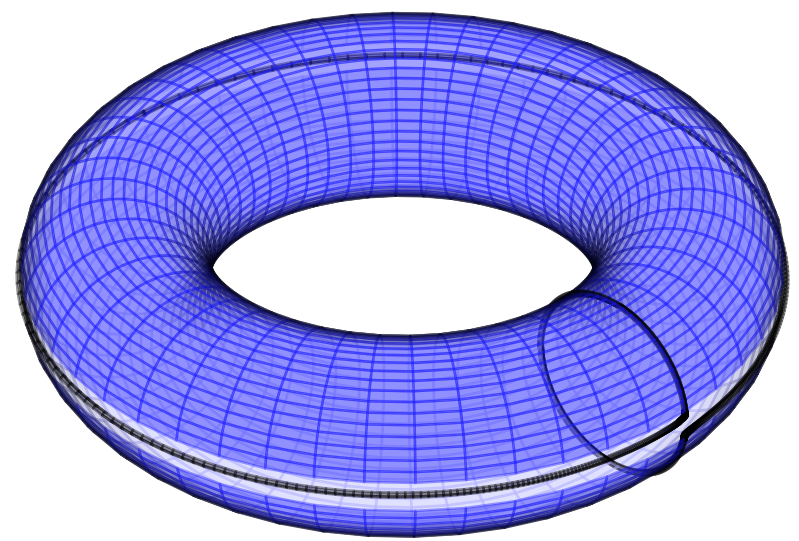

Figure 7: The annulus after surgery $(q=1)$

where $t \in(-\eta, \eta)$ parametrizes the flow direction and $s \in S^{1}$ is the parameter along $\mathfrak{c}$. $\gamma$ is parametrized by $\{0\} \times S^{1} \times\{0\}$. This gives a chart as in Proposition 2.2:

Lemma 5.1 The standard contact form $A$ in this chart satisfies

$$
A=d t+w d s, \quad d A=d w \wedge d s \quad \text { and } \quad A \wedge d A=d t \wedge d w \wedge d s .
$$

Proof If $g_{s}$ denotes the Riemannian metric at $(0, s) \in \Sigma$ and we write $(0, s, \theta)=$ $(x, u) \in S \Sigma$, then for a vector

$$
Z=a \frac{\partial}{\partial t}+b \frac{\partial}{\partial s}+c \frac{\partial}{\partial \theta}
$$

we have

$$
A_{(0, s, \theta)}(Z)=g_{s}(u, d \pi(Z))=g_{s}\left(u, a d \pi\left(\frac{\partial}{\partial t}\right)+b d \pi\left(\frac{\partial}{\partial s}\right)\right)=a+b g_{s}\left(u, \frac{\partial}{\partial s}\right)
$$

since $d \pi(\partial / \partial \theta)=0$.

Taking $S^{1}$ to have length $2 \pi$ we necessarily obtain $\|\partial / \partial s\|=\ell / 2 \pi$. Since a priori $A_{(t, s, \theta)}=d t+g d s+h d \theta$ with functions $g$ and $h$, this implies $A_{(t, s, \theta)}=d t+$ $\frac{\ell}{2 \pi} \cos \theta d s$, that is, $A=d t+w d s$. The other claims immediately follow.

\subsection{Goodman surgery and beyond}

While the Handel-Thurston surgery is described for a separating geodesic and hyperbolicity of the phase space in Theorem 1.12 uses a filling geodesic, neither of these is needed for the perpendicular vector field to the geodesic to define an $E$-transverse Legendrian knot; our surgery subsumes all these possibilities. Moreover, such surgeries 
can be carried out successively, using Legendrian knots from the preceding intermediate stage.

Goodman [26] gave surgeries inspired by those of Handel and Thurston but carried out in the vicinity of orbits (Figure 8, taken from [26], shows her Dehn torus as seen along the orbit in question). The salient point is that for every closed orbit of a suspension (of

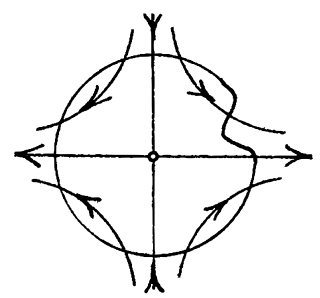

Figure 8: The Goodman surgery annulus (tilted segment in first quadrant)

a hyperbolic toral automorphism), Goodman produces an isotopic knot near which to perform Dehn surgery. In the contact category, geodesic flows (of hyperbolic surfaces) are the counterpart to her context, and they give rise to Legendrian knots in a similar manner:

Theorem 5.2 Consider the geodesic flow on the unit tangent bundle of a negatively curved surface. Then for each finite union of free homotopy classes of closed curves there exists an $E$-transverse Legendrian link that includes precisely one knot in each of the chosen homotopy classes.

Proof Since negative curvature implies hyperbolicity of the geodesic flow, each of the chosen homotopy classes contains a closed orbit of the geodesic flow. These are pairwise disjoint, and each of these is isotopic to a knot given by rotation of the tangent vector field by $-\pi / 2$, and these knots are pairwise disjoint. As before, they are Legendrian knots.

After choosing pairwise disjoint neighborhoods of these Legendrian knots, we can perform surgery in each of these as described in Section 2.

We remark that the Goodman surgery (unlike that presented by Handel and Thurston) can be carried out recursively; it applies near any closed orbit of any Anosov flow. In like manner, our surgery can be carried out repeatedly or simultaneously on finite collections of knots, that is, we have described surgery on $E$-transverse Legendrian links. 


\subsection{Transversality}

One may wonder whether our surgery can be applied in the following generality: Given a 3-manifold with a contact Anosov flow, is a knot always isotopic (or close) to an $E$-transverse Legendrian knot? Without " $E$-transverse" the answer is positive:

Proposition 5.3 (Geiges [23, Theorem 3.3.1]) A knot in a contact 3-manifold is isotopic to a $C^{0}$-close Legendrian knot.

It is, however, not readily apparent that one can arrange for the knot to be $E$-transverse. This calls attention to our transversality assumption, and it is well to note that while it may not be essential for performing our surgery, it is needed to establish that the resulting flow is an Anosov flow.

\section{Geometry of the Handel-Thurston surgery}

We return to the Handel-Thurston context of Section 5.1 and show that in this case we never get flows that are topologically orbit equivalent to an algebraic flow.

Definition 6.1 A 3-manifold is said to be Seifert-fibered if it admits a decomposition into a disjoint union of circles (the fibers) such that each fiber has a tubular neighborhood diffeomorphic (in a fiber-preserving way) to the torus $D^{2} \times S^{1}$ obtained from $D^{2} \times[0,1]$ by identifying $D^{2} \times\{0\}$ and $D^{2} \times\{1\}$ via a rational rotation.

We now recall the proof Handel and Thurston [28] that the flows thus obtained are not topologically orbit equivalent to an algebraic flow.

Theorem 6.2 When the surgery is carried out using a separating curve, the flow $\varphi_{h}^{t}$ in Theorem 4.3 is not topologically orbit equivalent to an algebraic Anosov flow. More strongly (see Tomter [51, page 419]), no finite cover of the surgered manifold $M$ from Definition 3.1 is a Seifert-fibered manifold (much less a sphere bundle) or a torus bundle over a circle.

Proof (Handel and Thurston [28]) We study finite covers of $M$ by examining their fundamental group. The two pertinent facts are

(1) The fundamental group of a torus bundle over a circle is solvable; thus we wish to show that $\pi_{1}(M)$ is not virtually solvable, that is, has no solvable finite-index subgroup. 
(2) The fundamental group of a Seifert-fibered manifold contains an infinite normal cyclic subgroup generated by a regular fiber (see Scott [47, page 432]); thus we want to show that no finite-index subgroup of $\pi_{1}(M)$ contains an infinite cyclic normal subgroup.

One observation that we will use for both of these items is the following.

Remark 6.3 If a group contains a finite-index subgroup $H$ and a free subgroup $F$, then $H$ contains a subgroup of $F$ that is isomorphic to $F$.

By the van Kampen Theorem (see, for example, Hatcher [30, Theorem 1.20]), we have

$$
\pi_{1}(M)=\pi_{1}\left(M_{1}\right)_{\pi_{1}\left(\partial M_{1}\right)}^{*} \pi_{1}\left(M_{2}\right)=\pi_{1}\left(M_{1}\right)_{\pi_{1}\left(\partial M_{2}\right)}^{*} \pi_{1}\left(M_{2}\right),
$$

using the isomorphism $F_{*}: \pi_{1}\left(\partial M_{1}\right) \rightarrow \pi_{1}\left(\partial M_{2}\right)$ induced by $F$ (as introduced in Definition 3.1).

Puncturing a surface of genus $g$ and retracting the remainder to its skeleton (a string of $2 g$ circles) shows that the fundamental group is a free group with $2 g$ generators. Thus, we see that $\pi_{1}\left(M_{i}\right)=F_{i} \oplus \mathbb{Z}$ for $i=1,2$, where $F_{1}$ and $F_{2}$ are free groups.

If $H<\pi_{1}(M)$ has finite index, then, as remarked above, it contains a free group inherited from $F_{1}$ or $F_{2}$, and since this holds recursively, $H$ is not solvable, and item (1) above is settled.

To settle item (2) suppose $\langle g\rangle<H$ is an infinite cyclic normal subgroup. This means that for every $h \in H$ there is a $p_{h} \in \mathbb{Z}$ such that $h g h^{-1}=g^{p_{h}}$. Clearly, $p_{h_{1} h_{2}}=p_{h_{1}} p_{h_{2}}$ for any $h_{1}, h_{2} \in H$, so $p_{\text {Id }}=1$ implies that for each $h \in H$ we have $p_{h} \in\{ \pm 1\}$ and $p_{h}=p_{h^{-1}}$. Thus, after possibly passing to the index-2 subgroup $\left\{h \in H \mid p_{h}=1\right\}$, we may assume without loss of generality that $H$ is in the centralizer of $g$, that is, $g h=h g$ for all $h \in H$.

As remarked above, there is a free group $F_{H} \subset F_{1} \cap H$ isomorphic to $F_{1}$ (we only need that it is large enough). We can write $g=w r^{k}$ with $w$ a word in generators of $F_{1}$ and $F_{2}$ only and $r$ the generator of the $S^{1}$-factor of $\pi_{1}\left(M_{1}\right)$ : writing one of the generators of $\pi_{1}\left(\partial M_{2}\right)$ as $\alpha_{a} r=a=\omega_{a} r^{\prime k}$ with $\alpha_{a} \in F_{1}, \omega_{a} \in F_{2}$ and $r^{\prime}$ the generator of the $S^{1}$-factor of $\pi_{1}\left(M_{2}\right)$, we find that any occurrence of $r \omega$ with $\omega \in F_{2}$ can be rewritten as $\alpha_{a}^{-1} \omega_{a} \omega \omega_{a}^{-1} \alpha_{a} r$; one applies this recursively to get the claim. We thus find that for any $h \in F_{H} \subset F_{1}$ we get

$$
w h r^{k}=w r^{k} h=g h=h g=h w r^{k},
$$


that is, $w h=h w$. But for $w \neq \mathrm{Id}$, this only holds when $h$ is a power of $w$. Since $F_{H}$ is not a subgroup of a cyclic group, we must have $g=r^{k}$ for some $k \in \mathbb{Z}$.

However, the same reasoning using $F_{2}$ shows that $g=r^{\prime \ell}$ for some $\ell \in \mathbb{Z}$, where $r^{\prime}$ is the generator of the $S^{1}$-factor of $\pi_{1}\left(M_{2}\right)$. This is incompatible with the earlier observation that $g=r^{k} \sim\left(s r^{\prime}\right)^{k}$, where $s$ represents the word in $F_{2}$ corresponding to the slope of the surgery-unless $s=\mathrm{Id}$ and the surgery is therefore trivial.

This implies item (2) above.

\section{Appendix A Anosov flows and Lyapunov-Lorentz metrics}

This appendix reproduces a way of expressing the Alexeev cone criterion for hyperbolicity in terms of Lorentz metrics that behave analogously to Lyapunov functions or metrics (see Barbot [5, page 16]).

Proposition A.1 A smooth flow $\varphi^{t}: M \rightarrow M$ of a 3-manifold $M$ is an Anosov flow if and only if there are two continuous Lorentz metrics $Q^{+}$and $Q^{-}$on $M$ and constants $a, b, c, T>0$ such that

(1) for all $v \in T_{x} M, t>T$, if $Q^{ \pm}(v)>0$ then $Q^{ \pm}\left(D_{x} \varphi^{ \pm t}(v)\right)>a e^{b t} Q^{ \pm}(v)$,

(2) $C^{+} \cap C^{-}=\varnothing$, where $C^{ \pm}$is the $Q^{ \pm}$-positive cone,

(3) $Q^{ \pm}(X)=-c$ where $X$ is the generating vector field,

(4) $D_{x} \varphi^{ \pm T}\left(\overline{C^{ \pm}(x)}\right) \backslash\{0\} \subset C^{ \pm}\left(\varphi^{ \pm T}(x)\right)$.

Proof If $\varphi^{t}$ is an Anosov flow we can choose disjoint cones around the strong stable and unstable directions, neither of which contains $X$. These define (up to a factor) the Lorentz metrics, and choosing $c=1$ fixes the metrics; we omit the details.

Assume now the above conditions for two continuous Lorentz metrics $Q^{ \pm}$and constants $a, b, c, T>0$. The cone fields $C^{ \pm}$induce fields $\mathcal{E}^{ \pm}$of ellipses in the projectivization $P T M$ of $T M$, and $\varphi^{t}$ acts on fields of ellipses by $\left(\varphi_{*}^{t} \mathcal{E}\right)(x):=$ $P D_{\varphi^{-t}(x)} \varphi^{t}\left(\mathcal{E}\left(\varphi^{-t}(x)\right)\right)$. Then

- condition (2) implies that $\mathcal{E}_{t}^{+}(x) \cap \mathcal{E}_{t}^{-}(x)=\varnothing$,

- condition (4) implies that $\overline{\mathcal{E}_{T}^{ \pm}(x)} \subset \operatorname{int} \mathcal{E}^{ \pm}(x)$.

If we endow each $\mathcal{E}^{ \pm}(x)$ with the Hilbert metric then this last property (strict nesting) implies that $D \varphi^{ \pm T}$ induces contractions $\mathcal{E}^{ \pm}(x) \rightarrow \mathcal{E}^{ \pm}\left(\varphi^{ \pm T}(x)\right)$ of the Hilbert metrics with a factor that can be chosen uniformly by compactness of $M$. Thus, the diameter of 
$\mathcal{E}_{t}^{ \pm}(x) \subset \mathcal{E}^{ \pm}(x)$ as measured by the Hilbert metric on $\mathcal{E}^{ \pm}(x)$ goes to 0 exponentially, so $\Delta^{ \pm}(x):=\bigcap_{t>T} \mathcal{E}_{t}^{ \pm}(x)$ are points, and $\Delta^{+}(x) \neq \Delta^{-}(x)$ for all $x \in M$ since $\mathcal{E}_{t}^{+}(x) \cap \mathcal{E}_{t}^{-}(x)=\varnothing$.

Clearly $\Delta^{ \pm}$define $\varphi^{t}$-invariant line fields $E^{ \pm}$, and since $X \notin C^{ \pm}$by condition (3), $\Delta^{+}(x) \neq X(x) \neq \Delta^{-}(x)$.

Now choose a continuous Riemannian metric on $M$ whose unit spheres intersect $E^{ \pm}$ in points for which $Q^{ \pm}=1$. Then condition (1) implies that $E^{ \pm}$are exponentially expanding and contracting, respectively, as required.

\section{Appendix B Hyperbolicity of complements of filling knots}

This appendix proves Theorem 1.12. Choosing $\gamma$ as in Theorem 1.12, $M_{\gamma}:=M \backslash U_{\gamma}$, the complement of a tubular neighborhood of $\gamma$ in $M$, is a manifold whose boundary is a 2-torus, and

Proposition B.1 $M_{\gamma}$ is irreducible (every embedded 2-sphere bounds a ball).

Proof To establish this we use that $M$ is itself irreducible:

Proposition B.2 (Hatcher [31, Proposition 1.12]) Compact connected Seifert-fibered manifolds are irreducible, except for $S^{1} \times S^{2}, S^{1} \tilde{\times} S^{2}, \mathbb{R} P^{3} \# \mathbb{R} P^{3}$.

If $S^{2}$ is embedded into $M_{\gamma}$ and hence into $M$, then it bounds a ball $B \subset M$. Since $S^{2} \cap \gamma=\varnothing$ we have either $\gamma \subset B$ or $B \cap \gamma=\varnothing$. Since $\gamma$ projects to a filling geodesic, it does not lie in a ball, so $B \cap \gamma=\varnothing$ and $B \subset M_{\gamma}$, as required.

Since $M_{\gamma}$ has nontrivial fundamental group, it is a Haken manifold:

Theorem B.3 (Matignon [41, page 14], Thurston [49, page 72]) An irreducible 3-manifold with boundary is either a Haken manifold or the 3-ball.

Moreover, we have the following.

Proposition B.4 $M_{\gamma}$ is atoroidal.

Definition B.5 (Hatcher [31]) A 3-manifold $M$ is said to be atoroidal if every incompressible torus in $M$ is boundary-parallel. Here, a 2-torus in $M$ is said to be 
- compressible if every essential circle (that is, one that bounds no disk in the 2 -torus) on it bounds a disk (a "compression disk") in $M$.

- boundary-parallel if there is an isotopy from it to $\partial M$, that is, there is an embedded $\mathbb{T}^{2} \times[0,1]$ such that $\mathbb{T}^{2} \times\{0\}$ parametrizes the given torus and $\mathbb{T}^{2} \times\{1\}$ a boundary component.

This implies Theorem 1.12:

Theorem B.6 (Hyperbolization Theorem, Thurston [50], Kapovich [37], Otal [43]) The interior of a compact atoroidal Haken 3-manifold with torus boundary admits a complete hyperbolic Riemannian metric (that is, with sectional curvature -1) of finite volume.

It remains to prove Proposition B.4.

Proof of Proposition B.4 Suppose that $\mathbb{T}$ is an incompressible 2-torus in $M_{\gamma}$. We need to show that $\mathbb{T}$ is boundary-parallel. Our first step is to show

Claim B.7 $\mathbb{T}$ is compressible in $M$.

Proof Otherwise the isotopy extension lemma (Hirsch [32, Theorem 8.1.3, page 180]) gives is an isotopy of $M$ that produces one or the other of the scenarios in the following theorem.

Theorem B.8 (Waldhausen [52, Satz 2.8]) An incompressible torus in an $S^{1}$-bundle is isotopic to one of the following: A union of fibers or a torus $T$ such that $\pi \uparrow T$ (the base-point projection) is a covering map.

The second one is impossible because it means that $\Sigma$ is covered by a 2-torus although $\Sigma$ has higher genus. Thus, instead, we have an isotopy of $M$ that presents $\mathbb{T}$ as a union of fibers, which means that under $\pi$ it projects to a curve in $\Sigma$ that is (by incompressibility of $\mathbb{T}$ ) not null-homotopic and yet disjoint from $\mathfrak{c}=\pi(\gamma)$ (since $\mathbb{T} \cap \gamma=\varnothing)$, contrary to the assumption that $\mathfrak{c}$ fills $\Sigma$.

The second step towards $\mathbb{T}$ being boundary-parallel is the following lemma.

Lemma B.9 $\mathbb{T}$ bounds a solid torus $\tau \subset M$ that contains $\gamma$. 
Proof (See also Hatcher [31, Section 1.2].) That $\gamma$ must lie in any such torus is clear: $\tau \cap \gamma \neq \varnothing$ (otherwise $\mathbb{T}$ is compressible in $M_{\gamma}$ ), but then $\mathbb{T} \subset M_{\gamma} \Rightarrow \mathbb{T} \cap \gamma=\varnothing \Rightarrow$ $\gamma \subset \tau$ by connectedness.

Compressibility of $\mathbb{T}$ means that there is a compression disk $D$. Compressing $D$ pinches $\mathbb{T}$ off to a 2-sphere, which by irreducibility (see Waldhausen [52, Lemma 2.7] and Hatcher [31, Proposition 1.12]), bounds a ball $B$. We presently show that $B \cap D=$ $\varnothing$, so uncompressing again and adding the compression disk deforms $B$ into the desired solid torus $\tau$ by attaching a handle to the bounding sphere from the outside.

To see that $B \cap D=\varnothing,{ }^{6}$ note that otherwise $D \subset B$ and hence $\mathbb{T} \subset B$, so $\mathbb{T}$ lifts to the universal cover $\tilde{M}$ of $M$. Since $\mathbb{T}$ is incompressible in $M_{\gamma}$, it is incompressible in any cover of $M_{\gamma}$ to which it lifts, so it is incompressible in $\tilde{M} \backslash \tilde{\gamma}$, where $\tilde{\gamma}$ denotes the total preimage of $\gamma$ under the universal covering map. This is impossible: $\tilde{M} \backslash \tilde{\gamma}$ is homeomorphic to $\left(\mathbb{R}^{2} \backslash X\right) \times \mathbb{R}$ for some infinite discrete set $X \subset \mathbb{R}^{2}$ (because the geodesic flow is globally product-covered), so its fundamental group is free, which precludes the presence of an incompressible torus (an embedded torus is incompressible if and only if its fundamental group injects).

The final step is that $\gamma$ lies in $\tau$ in such a way as to allow the desired homotopy from $\mathbb{T}$ to the boundary of a tubular neighborhood of $\gamma$. To that end let $\beta$ be a simple closed curve representing the core curve of $\tau$, that is, a curve such that arbitrarily small tubular neighborhoods are isotopic to $\tau$. Then "boundary-parallel" and hence Proposition B.4 follows from the next lemma.

\section{Lemma B.10 $\gamma$ and $\beta$ are isotopic in $\tau$.}

Proof (Fenley-Walsh) The main step is to show that these curves are homotopic. By assumption $\gamma$ meets any candidate for a compression disk, so $\gamma$ represents a nontrivial element of the fundamental group of $\tau$, that is, $\gamma$ is homotopic to $\beta^{n}$ for some $n \in \mathbb{Z}$. Homotopy follows once we show that $|n|=1$.

To this end, denote by $g$ the deck transformation associated to $\gamma$ with $g(\tilde{\gamma})=\tilde{\gamma}$, and let $h$ be the deck transformation corresponding to $\beta$ and such that $h^{n}=g$. To find an $h$-invariant orbit, consider the lift of the stable foliation to $\tilde{M}$; its leaf space is homeomorphic to $\mathbb{R}$ (geodesic flows are $\mathbb{R}$-covered), and $g$ does not act freely on it since it preserves $\tilde{\gamma}$ and hence its stable leaf. Therefore, $h$ does not act freely either, that is, it preserves a leaf and hence a flow-line $\tilde{\eta}$ in it. Since $g=h^{n}$ preserves both $\tilde{\gamma}$ and $\tilde{\eta}$, the closed orbits $\gamma$ and $\eta$ in $M=S \Sigma$ to which they project represent the same

${ }^{6}$ This paragraph is due to Calegari. 
element of the fundamental group and are freely homotopic. The homotopy projects to a free homotopy in $S$ of $\mathfrak{c}=\pi(\gamma)$ and a geodesic $\mathfrak{e}$ traversed $n$ times. Since closed geodesics in $S$ are the unique minimizers in their homotopy class, $\mathfrak{c}=\mathfrak{e}$ as geodesics. By assumption, $\mathfrak{c}$ is not the same geodesic traversed more than once, and $|n|=1$.

To see that $\gamma$ is isotopic to $\beta$, note that, since $\gamma$ projects to a closed geodesic, $\tilde{\gamma}$ is not knotted in $\tilde{M}$ and hence not knotted in $\tilde{\tau}$, an infinite cylinder. Thus, $\gamma$ is not knotted in $\tau$.

Acknowledgements We feel deep gratitude to Thierry Barbot, Thomas Barthelmé, Danny Calegari, Sérgio Fenley, Jean-Pierre Otal and Genevieve Walsh for sharing their insights with us. Barbot provided the particularly elegant proof of hyperbolicity of the surgered flow and allowed us to reproduce the requisite background in Appendix A, the consultations with Calegari, Fenley, Otal and Walsh enabled us to produce here a proof of hyperbolicity of the surgered manifold (Appendix B), and conversations with Barthelmé were an essential ingredient while writing this paper. It is furthermore a pleasure to acknowledge the congenial hospitality and working conditions of the Collège Doctoral Européen in Strasbourg as well as the Centre International de Rencontres Mathématiques in Luminy. This work was partially supported by the Committee on Faculty Research Awards of Tufts University.

\section{References}

[1] E Artin, Ein mechanisches System mit quasiergodischen Bahnen, Abh. Math. Semin. Univ. Hamb. 3 (1924) 170-175

[2] T Barbot, Caractérisation des flots d'Anosov en dimension 3 par leurs feuilletages faibles, Ergodic Theory Dynam. Systems 15 (1995) 247-270 MR1332403

[3] T Barbot, Generalizations of the Bonatti-Langevin example of Anosov flow and their classification up to topological equivalence, Comm. Anal. Geom. 6 (1998) 749-798 MR1652255

[4] T Barbot, Plane affine geometry and Anosov flows, Ann. Sci. École Norm. Sup. 34 (2001) 871-889 MR1872423

[5] T Barbot, De l'hyperbolique au globalement hyperbolique, Habilitation à diriger des recherches, Université Claude Bernard de Lyon (2005) Available at http:// www . umpa.ens-lyon.fr/ barbot/HABILITATION/memoireCRY .pdf

[6] T Barthelmé, A new Laplace operator in Finsler geometry and periodic orbits of Anosov flows, PhD thesis, Université de Strasbourg (2012) arXiv: 1204.0879

[7] T Barthelmé, S R Fenley, Knot theory of R-covered Anosov flows: Homotopy versus isotopy of closed orbits arXiv:1208.6487 
[8] Y Benoist, P Foulon, F Labourie, Flots d'Anosov à distributions de Liapounov différentiables, I, Ann. Inst. H. Poincaré Phys. Théor. 53 (1990) 395-412 MR1096099

[9] Y Benoist, P Foulon, F Labourie, Flots d'Anosov à distributions stable et instable différentiables, J. Amer. Math. Soc. 5 (1992) 33-74 MR1124979

[10] C Bonatti, R Langevin, Un exemple de flot d'Anosov transitif transverse à un tore et non conjugué à une suspension, Ergodic Theory Dynam. Systems 14 (1994) 633-643 MR1304136

[11] D Calegari, The geometry of $\mathbb{R}$-covered foliations, Geom. Topol. 4 (2000) 457-515 MR1800151

[12] D Calegari, Filling geodesics and hyperbolic complements, Geometry and the Imagination blog (2012) Available at http://lamington.wordpress.com/2012/02/11/ filling-geodesics-and-hyperbolic-complements

[13] J P Christy, Anosov flows on three-manifolds (topology, dynamics), PhD thesis, University of California, Berkeley (1984) MR2633722

[14] Y Fang, Thermodynamic invariants of Anosov flows and rigidity, Discrete Contin. Dyn. Syst. 24 (2009) 1185-1204 MR2505698

[15] S R Fenley, Anosov flows in 3-manifolds, Ann. of Math. 139 (1994) 79-115 MR1259365

[16] S R Fenley, Quasigeodesic Anosov flows and homotopic properties of flow lines, J. Differential Geom. 41 (1995) 479-514 MR1331975

[17] S R Fenley, Foliations, topology and geometry of 3-manifolds: $\mathbb{R}$-covered foliations and transverse pseudo-Anosov flows, Comment. Math. Helv. 77 (2002) 415-490 MR1933786

[18] S Fenley, Ideal boundaries of pseudo-Anosov flows and uniform convergence groups with connections and applications to large scale geometry, Geom. Topol. 16 (2012) 1-110 MR2872578

[19] R Feres, A Katok, Invariant tensor fields of dynamical systems with pinched Lyapunov exponents and rigidity of geodesic flows, Ergodic Theory Dynam. Systems 9 (1989) 427-432 MR1016661

[20] P Foulon, Entropy rigidity of Anosov flows in dimension three, Ergodic Theory Dynam. Systems 21 (2001) 1101-1112 MR1849603

[21] P Foulon, B Hasselblatt, Zygmund strong foliations, Israel J. Math. 138 (2003) 157169 MR2031955

[22] J Franks, B Williams, Anomalous Anosov flows, from: "Global theory of dynamical systems", (Z Nitecki, C Robinson, editors), Lecture Notes in Math. 819, Springer, Berlin (1980) 158-174 MR591182

[23] H Geiges, An introduction to contact topology, Cambridge Studies in Advanced Mathematics 109, Cambridge Univ. Press (2008) MR2397738 
[24] É Ghys, Flots d'Anosov sur les 3-variétés fibrées en cercles, Ergodic Theory Dynam. Systems 4 (1984) 67-80 MR758894

[25] É Ghys, Flots d'Anosov dont les feuilletages stables sont différentiables, Ann. Sci. École Norm. Sup. 20 (1987) 251-270 MR911758

[26] S Goodman, Dehn surgery on Anosov flows, from: "Geometric dynamics", (J Palis, Jr, editor), Lecture Notes in Math. 1007, Springer, Berlin (1983) 300-307 MR1691596

[27] U Hamenstädt, Regularity of time-preserving conjugacies for contact Anosov flows with $C^{1}$-Anosov splitting, Ergodic Theory Dynam. Systems 13 (1993) 65-72 MR1213079

[28] M Handel, W P Thurston, Anosov flows on new three manifolds, Invent. Math. 59 (1980) 95-103 MR577356

[29] B Hasselblatt, Hyperbolic dynamical systems, from: "Handbook of dynamical systems 1A", (B Hasselblatt, A Katok, editors), North-Holland, Amsterdam (2002) 239-319 MR1928520

[30] A Hatcher, Algebraic topology, Cambridge Univ. Press (2002) MR1867354

[31] A Hatcher, Notes on basic 3-manifold topology (2007) Available at http:// www . math. cornell . edu/ hatcher/3M/3Mfds.pdf

[32] M W Hirsch, Differential topology, Graduate Texts in Mathematics 33, Springer, New York (1976) MR0448362

[33] H Hofer, Pseudoholomorphic curves in symplectizations with applications to the Weinstein conjecture in dimension three, Invent. Math. 114 (1993) 515-563 MR1244912

[34] E Hopf, Statistik der geodätischen Linien in Mannigfaltigkeiten negativer Krümmung, Ber. Verh. Sächs. Akad. Wiss. Leipzig 91 (1939) 261-304 MR0001464

[35] S Hurder, A Katok, Differentiability, rigidity and Godbillon-Vey classes for Anosov flows, Inst. Hautes Études Sci. Publ. Math. (1990) 5-61 MR1087392

[36] M Kanai, Geodesic flows of negatively curved manifolds with smooth stable and unstable foliations, Ergodic Theory Dynam. Systems 8 (1988) 215-239 MR951270

[37] M Kapovich, Hyperbolic manifolds and discrete groups, Progress in Mathematics 183, Birkhäuser, Boston, MA (2001) MR1792613

[38] A Katok, B Hasselblatt, Introduction to the modern theory of dynamical systems, Encyclopedia of Mathematics and its Applications 54, Cambridge Univ. Press (1995) MR1326374

[39] C Liverani, On contact Anosov flows, Ann. of Math. 159 (2004) 1275-1312 MR2113022

[40] J Martinet, Formes de contact sur les variétés de dimension 3, from: "Proceedings of Liverpool Singularities Symposium II", (C T C Wall, editor), Lecture Notes in Math. 209, Springer, Berlin (1971) 142-163 MR0350771 
[41] D Matignon, Topologie en basse dimension: Remplissages de Dehn et théorie des nœuds, Habilitation à diriger des recherches, Université d'Aix-Marseille I (2004) Available at http://www.cmi.univ-mrs.fr/ matignon/travaux-pdf/HDR.pdf

[42] Y Mitsumatsu, Anosov flows and non-Stein symplectic manifolds, Ann. Inst. Fourier (Grenoble) 45 (1995) 1407-1421 MR1370752

[43] J-P Otal, Le théorème d'hyperbolisation pour les variétés fibrées de dimension 3, Astérisque 235 (1996) x+159 MR1402300

[44] C Petronio, J Porti, Negatively oriented ideal triangulations and a proof of Thurston's hyperbolic Dehn filling theorem, Expo. Math. 18 (2000) 1-35 MR1751141

[45] J F Plante, Anosov flows, Amer. J. Math. 94 (1972) 729-754 MR0377930

[46] J F Plante, W P Thurston, Anosov flows and the fundamental group, Topology 11 (1972) 147-150 MR0295389

[47] P Scott, The geometries of 3-manifolds, Bull. London Math. Soc. 15 (1983) 401-487 MR705527

[48] W P Thurston, Three-manifolds, foliations and circles I arXiv:math/9712268

[49] W P Thurston, The geometry and topology of three-manifolds (1980) Available at http://library.msri.org/books/gt3m/

[50] W P Thurston, Three-dimensional manifolds, Kleinian groups and hyperbolic geometry, Bull. Amer. Math. Soc. 6 (1982) 357-381 MR648524

[51] P Tomter, Anosov flows on infra-homogeneous spaces, from: "Global analysis", Proc. Sympos. Pure Math. 14, Amer. Math. Soc. (1970) 299-327 MR0279831

[52] F Waldhausen, Eine Klasse von 3-dimensionalen Mannigfaltigkeiten I, Invent. Math. 3 (1967) 308-333 MR0235576

Institut de Recherche Mathematique Avancée, UMR 7501 du Centre National de la Recherche Scientifique, 7 Rue René Descartes, 67084 Strasbourg Cedex, France

Centre International de Rencontres Mathématiques, 163 Avenue de Luminy

Case 916, 13288 Marseille Cedex 9, France

Department of Mathematics, Tufts University

Medford, MA 02155, USA

foulon@math.u-strasbg.fr, foulon@cirm.univ-mrs.fr,

Boris.Hasselblatt@tufts.edu

Proposed: Danny Calegari

Seconded: Ronald Stern, Leonid Polterovich
Received: 1 February 2012

Revised: 10 February 2013 